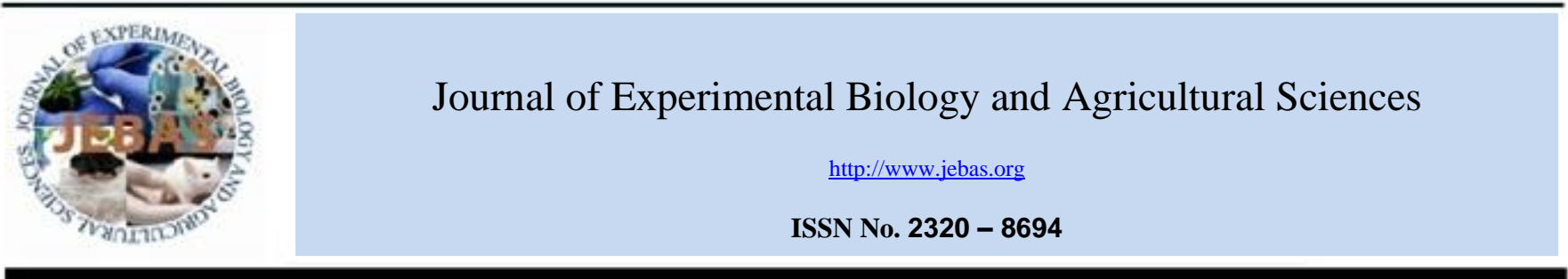

\title{
CHARACTERIZATION OF ENDOPHYTIC BACTERIA WITH PLANT GROWTH PROMOTING ACTIVITIES ISOLATED FROM SIX MEDICINAL PLANTS
}

\author{
Sangeeta Panigrahi ${ }^{1}$, Debasis Dash ${ }^{1}$, Chandi Charan Rath ${ }^{2 *}$ \\ ${ }^{1}$ Department of Botany, College of Basic Science and Humanities, Orissa University of Agriculture and Technology (OUAT), Bhubaneswar, 751003, India \\ ${ }^{2}$ PG Department of Life Sciences, Rama Devi Women's University, Bhubaneswar, 751022, India
}

Received - July 30, 2018; Revision - September 05, 2018; Accepted - September 28, 2018

Available Online - October 5, 2018

DOI: http://dx.doi.org/10.18006/2018.6(5).782.791

KEYWORDS
Medicinal plants
Endophytic bacteria
Plant growth promotion
IAA
Siderophore production
Phosphate solubilization

\begin{abstract}
The present investigation aimed to isolate and characterize endophytic bacteria from six selected medicinal plants (Ocimum sanctum, Aegle marmelos, Cinnamomum cassia, Azadirachta indica, Calotropis procera and Rauwolfia serpentina) with plant growth promoting activities. Total 38 endophytic bacteria were isolated from the studied plant species by following standard microbiological culture methods. All the isolated bacteria were screened for the production of hydrolyzing enzyme and result of study revealed that maximum isolates have positive catalytic activity and this was followed by amylase, lipase and protease activities. While studying the antibacterial activity of the isolates, it was observed that fifteen isolates showed moderate to higher antibacterial activities against Escherichia coli, Pseudomonas aeruginosa \& Staphylococcus aureus. Studies on plant growth promoting activity suggested that $8,12 \& 6$ isolates were positive for IAA production, siderophore production and phosphate solubilization activity respectively. Further, eight $(21.05 \%)$ isolates were found positive for ammonia production. Through this investigation, endophytic bacteria isolated from medicinal plants with plant growth promoting activities were reported, that can be further exploited for sustainable agriculture, with detail scientific investigation.
\end{abstract}

* Corresponding author

E-mail: chandicharanrath@yahoo.com (Chandi Charan Rath)

Peer review under responsibility of Journal of Experimental Biology and Agricultural Sciences.

Production and Hosting by Horizon Publisher India [HPI] (http://www.horizonpublisherindia.in/).

All rights reserved.
All the article published by Journal of Experimental Biology and Agricultural Sciences is licensed under a Creative Commons Attribution-NonCommercial 4.0 International License Based on a work at www.jebas.org.

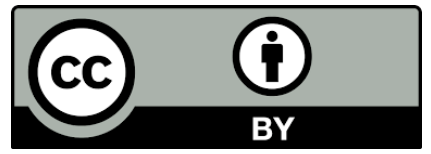




\section{Introduction}

Medicinal plants have been gaining global attention owing to the fact that the herbal drugs are cost effective, easily available and with negligible side effects. These plants harbor an untapped source of bioactive metabolites and large number of microorganisms, more specifically bacteria and fungi, called endophytes. Endophytes are the microorganisms which reside within the plant tissue without showing any overt negative effect. Nearly all plant species exist in the earth harbor one or more endophytic microorganisms (Strobel et al., 2004; Andreote et al., 2014). To survive in the plant tissue, these endophytes decompose plant metabolites and obtain nutrition and energy. Due to long term co-evolution of endophytes with their host plant, these endophytes have adapted to the special microenvironments by uptake of plant genome through genetic variation (Germaine et al., 2004). Colonization of endophytes in the host plant have number of benefits such as increased resistance to pathogens, herbivores, flooding stress, drought stress as well as enhanced competitive abilities (Mastretta et al., 2006; Taghvi et al., 2009). Not only endophytes, plants also remains beneficial from the presence of endophytes, these endophytes release antibiotics or hydrolytic enzymes which prevent colonization of microbial plant pathogens as well as prevent insect nematodes from being infecting the host plant also (Azevedo et al., 2000). By the process of induced systemic resistance, endophytes release metabolites which activate the host defence mechanism against other pathogens (Kloepper \& Ryu, 2006). Recently, endophytic bacteria have gained enough attention of both academicians and researchers because of their intimate relationship with plants that result in production of a variety of bioactive compounds. Several compounds derived from endophytic bacteria have great application value in developing medicine and they also act as biocontrol agents (Gunatilaka, 2006). In addition, endophytic bacteria promote plant growth by several mechanisms such as production of phytohormones (Lee et al., 2004), nitrogen fixation (Compant et al., 2005), phosphate solubilization (Wakelin et al., 2004), synthesis of siderophores ( Lodewyckx et al., 2002) etc. By considering the above said beneficial effects, present study has been carried out to isolate and characterize bacterial endophytes from selected medicinal plants and to evaluate their potential with respect to plant growth promotion (PGP) and antibacterial activities.

\section{Materials and Methods}

\subsection{Media and Chemical used}

Nutrient agar, Nutrient broth, Pikovskaya's agar, Chrome Azurol $\mathrm{S}$ (CAS) agar, Salkowski reagent $\left(0.1 \mathrm{ml}\right.$ of $0.5 \mathrm{M} \mathrm{FeCl}_{3}$ in $50 \mathrm{ml}$ of $35 \% \mathrm{HClO} 4)$, Nessler's reagent, Tween20, Sodium hypochlorite, L- Tryptophan, Orthophosphoric acid were procured from HiMedia Pvt. Ltd. Mumbai, India and prepared as per manufacturer's instructions.

\subsection{Collection of sample}

Healthy, mature and disease-free twigs of all selected medicinal plants (Ocimum sanctum, Azadirachta indica, Aegle marmelos, Cinnamomum cassia, Calotropis procera \& Rauwolfia serpentina) were collected from the botanical garden of College of Basic Science and Humanities, OUAT, kept in sterile sampling bags and brought to the laboratory for processing.

\subsection{Sample processing}

Plant parts like leaf, stem and petiole of all the sample plant twigs were washed in running tap water for $15 \mathrm{~min}$ and it was followed by washing in Tween 20 detergent for $1 \mathrm{~min}$ and finally rinsed with sterile distilled water for 3 times to remove the debris, soil particles and microorganisms adhered in the surfaces.

\subsection{Surface sterilization of explant \& effectiveness of surface sterilization}

In order to remove the epiphytic organism, surface sterilization process was performed by using different surface sterilants (Kumar et al., 2016). Leaf, stem and petiole were dissected from the twigs, by a sterilized blade under aseptic condition and were immersed in $70 \%$ ethanol for $2 \mathrm{~min}$, followed by 2 times rinse in sterile distilled water, this is repeated twice with intermittent treatment in $4 \%$ sodium hypochlorite for $5 \mathrm{~min}$ followed by 2 times rinse in sterile distilled water. The explants were rinsed with sterile distilled water 4-5 times and blot dried, on a sterilized blotting paper. To ensure, effective surface sterilization, $1 \mathrm{ml}$ of the finally rinsed sterile distilled water was cultured on Nutrient Agar plates, to check the presence of any bacteria. All the operations were carried out aseptically in a laminar flow hood.

\subsection{Isolation and purification of endophytic bacteria}

Surface sterilized leaf, stem and petiole of all the sample plants were cut into small pieces (1-3 mm long). The petiole \& stem were placed inverted with exposed part onto the media plates. Leaves were cut from the margin and were also placed onto the Nutrient Agar plates and were sealed with parafilm followed by the incubation at $37^{\circ} \mathrm{C}$ for 1-7 days to allow the growth of endophytic bacteria and were checked for every $24 \mathrm{hr}$ for counting (Pavlo et al., 2011). Colonies which appeared from the margins of the inoculated sample parts with different morphology were selected and pure cultured by repeated streaking method on Nutrient agar media plates and the pure cultures obtained were preserved at $4^{\circ} \mathrm{C}$ on Nutrient agar slants for further use. 


\subsection{Phenotypic characterization of the isolates}

Isolated endophytic bacteria were phenotypically characterized for growth characteristics on Nutrient agar, colony morphology and Grams reaction by using standard procedures in accordance with Holt et al. (1994) and Mbai et al. (2013)

\subsection{Biochemical characterization of the isolates}

All the isolates were screened for utilization of different sugars as source of carbon, $\mathrm{H}_{2} \mathrm{~S}$ production, Indole production, motility test, Urea and citrate utilization, through an array of biochemical tests proposed by Cappucino \& Sherman (2002).

\subsection{Enzyme test}

The isolates were studied for different extra cellular enzymatic activities like catalase, amylase, protease and lipase through substrate level plate assay method proposed by Amaresan et al. (2014).

\subsection{Antibacterial activity of bacterial endophytes}

Antibacterial activities of the isolates were tested using culture filtrates through disc diffusion method and spot inoculation method. Endophytic bacterial isolates were cultured separately on Nutrient agar (NA) plates and in conical flasks containing Nutrient broth at $37^{\circ} \mathrm{C}$ for $24 \mathrm{hrs}$. The broth culture was centrifuged at 5000rpm for $10 \mathrm{~min}$, and the supernatant was used for antibacterial assay. Overnight freshly grown cultures (in Nutrient broth) of the test organisms viz. S. aureus, E. coli and $P$. aeruginosa were seeded on NA plates with the help of a sterilized cotton swab separately. Sterilized filter paper discs of $5 \mathrm{~mm}$ diameter were placed over pathogen seeded NA plates, onto which $10 \mu 1$ of culture supernatant was added and dried under laminar airflow. Similarly, isolated endophytes were spot inoculated on the pathogen seeded plates with the help of an inoculating loop. Plates were incubated at $37^{\circ} \mathrm{C}$ overnight and observed for zone of inhibition around the discs and colonies as an indicative of antibacterial activities.

\subsection{Plant Growth Promoting Activity Tests}

All the isolates were qualitatively characterized for PGP activities like IAA production, phosphate solubilization, siderophore and ammonia production by following the standard protocols.

\subsubsection{IAA Production}

IAA Produced by the isolates was determined by following the methods given by Patten \& Glick (2002). Briefly $2 \mathrm{ml}$ of $24 \mathrm{hr}$ old broth culture of the isolate (Nutrient broth supplemented with $0.2 \%$ L- Tryptophan) was taken, to which 2 drops of Orthophosphoric acid and $4 \mathrm{ml}$ of Salkowski reagent was added and incubated in dark at room temperature $\left(25^{\circ} \mathrm{C}\right)$ for $30 \mathrm{~min}$. Development of pink color after the incubation period is the indicative of IAA production by a particular isolate. The above procedure was followed for all the isolates.

\subsubsection{Phosphate solubilization}

Phosphate solubilization activity was assayed by growing the culture on Pikovskaya's agar plate and incubating the plate at $37^{\circ} \mathrm{C}$ for $48-72 \mathrm{hr}$ as described by Jena \& Rath (2016). Formation of the clear halo zone around the colony indicates the solubilization of phosphate due to the utilization of tri-calcium phosphate present in the medium. Phosphate Solubilizing Index (PSI) of a positive isolate was determined using the formula presented below;

PSI $=($ colony diameter + halo zone diameter $) /$ Colony diameter

\subsubsection{Siderophore production}

Siderophore production by the isolates was studied following the method of Rashid et al. (2012). The isolates were spot inoculated on Chrome Azurol S (CAS) agar media and incubated at $37^{\circ} \mathrm{C}$ for 5 days. The appearance of orange yellow halo zone around the colonies was considered positive for the presence of siderophores. Siderophore production index (SI) was calculated using the formula as given below;

$$
\begin{gathered}
\mathrm{SI}=(\text { Colony diameter }+ \text { orange yellow halo zone diameter }) / \\
\text { Colony diameter }
\end{gathered}
$$

\subsubsection{Ammonia production}

All the bacterial isolates were tested for the production of ammonia following Nessler's reagent Method (Rashid et al. 2012).

\section{Results}

\subsection{Effectiveness of surface sterilization}

Two different methods were employed i.e., by culturing $1 \mathrm{ml}$ of aliquots of final rinsed water of each sample and by imprinting the surface sterilized tissues on nutrient agar plates and further incubating the plates at $37^{\circ} \mathrm{C}$ for 7 days. Both the surface sterilization methods were found to be satisfactory as no microbial growth appeared on these cultured plates. Hence, all the isolated organisms were considered as endophytic bacteria.

\subsection{Isolation and purification of endophytic bacteria}

Total 38 endophytic bacteria were isolated from surface sterilized stem, leaf and petioles of six selected medicinal plants $(O$. sanctum, A. marmelos, C. cassia, A. indica, C. procera and $R$. 
serpentina) by culturable methods on nutrient agar media and pure cultured. Yield of endophytic bacteria and their colonization frequency from different parts of selected plants are presented in Table 1. Maximum numbers of bacteria were isolated from $C$. procera.

\subsection{Characterization of endophytic bacteria}

All the isolates were further characterized by Gram's reaction, of which $25(65.78 \%$ ) found to be gram negative rods, $8(21.05 \%)$ were gram positive bacilli and $5(13.15 \%)$ were found to be gram positive cocci. Further, among the isolates, 24 isolates $(63.15 \%)$ showed motility in Mannitol motility agar whereas 14 (36.84\%) of the isolates revealed to be non-motile in the same medium. All the isolates utilized glucose, sucrose and lactose oxidatively when tested on Triple Sugar Iron agar (TSI) medium. Only seven isolates produced both acid and gas while the other 31 isolates produced only acid when tested on the same medium. Likewise, out of 38 isolates 26 and 21 isolates were positive for utilization of citrate and urea respectively, when grown on Simmons' citrate agar medium and Urea agar base supplemented with $40 \%$ filter sterilized urea separately. All the 38 isolates were negative for $\mathrm{H}_{2} \mathrm{~S}$ and Indole production. All the data are presented in Table 2. Based on morphology and biochemical characters, the gram +ve rods and gram -ve rods were tentatively identified as Bacillus and Pseudomonas sps. respectively (Collins \& Lyne 1970).

\subsection{Hydrolyzing enzyme activities}

All the endophytic bacterial isolates were subjected to evaluate the production of hydrolytic enzymes like catalase, amylase, lipase and protease. Among the bacterial isolates 35(92.10\%) were positive for catalase activity, rest 3 (CL01, CS01 \& CS07) showed negative response to catalase activity. Similarly, $27(71.05 \%)$ and $18(47.36 \%)$ of the isolates were positive for extracellular amylase and lipase activities respectively. Only four isolate (OS02, CL02, CS08 \& CSLS17) were found to be positive for protease activity respectively when studied through plate assay method as shown in Figure 1.

\subsection{Screening for antibacterial activity}

All the 38 isolates were screened against 3 test organisms viz. Pseudomonas aeruginosa, Escherichia coli and Staphylococcus aureus by spot inoculation method and disc diffusion Method. Out of 38 endophytic bacterial isolates, 15 (39.47\%) showed antibacterial activities against one or other test organism represented in Table 3. Out of 15 bacteria, 12 isolates showed inhibitory activity against $E$. coli and $S$. aureus whereas, 5 isolates showed activity against $P$. aeruginosa. Three isolates (OS03, OS01, OL01) showed positive activity against all the three test organisms.
Table 1 Colonization frequency of endophytic bacteria from six medicinal plants

\begin{tabular}{|c|c|c|c|c|}
\hline $\begin{array}{c}\text { Name of the } \\
\text { plant }\end{array}$ & $\begin{array}{l}\text { Plant } \\
\text { parts } \\
\text { used }\end{array}$ & $\begin{array}{c}\text { No. of } \\
\text { segments } \\
\text { inoculated }\end{array}$ & $\begin{array}{c}\text { No. of } \\
\text { isolates } \\
\text { Recovered }\end{array}$ & $\begin{array}{c}\% \text { frequency } \\
\text { of } \\
\text { colonization }\end{array}$ \\
\hline \multirow{3}{*}{$\begin{array}{l}\text { Ocimum } \\
\text { sanctum }\end{array}$} & Leaf & 15 & 1 & 6.66 \\
\hline & Stem & 15 & 3 & 20.00 \\
\hline & Petiole & 15 & 2 & 13.33 \\
\hline \multirow{3}{*}{$\begin{array}{l}\text { Aegle } \\
\text { marmelos }\end{array}$} & Leaf & 15 & 1 & 6.66 \\
\hline & Stem & 15 & 2 & 13.33 \\
\hline & Petiole & 15 & 1 & 6.66 \\
\hline \multirow{2}{*}{$\begin{array}{l}\text { Calotropis } \\
\text { procera }\end{array}$} & Leaf & 20 & 4 & 20.00 \\
\hline & Stem & 20 & 17 & 85.00 \\
\hline \multirow{2}{*}{$\begin{array}{l}\text { Rawolfia } \\
\text { serpentina }\end{array}$} & Leaf & 20 & 0 & 0.00 \\
\hline & Stem & 20 & 4 & 20.00 \\
\hline \multirow{3}{*}{$\begin{array}{l}\text { Cinnaтотит } \\
\text { cassia }\end{array}$} & Leaf & 15 & 0 & 0.00 \\
\hline & Stem & 15 & 0 & 0.00 \\
\hline & Petiole & 15 & 0 & 0.00 \\
\hline \multirow{3}{*}{$\begin{array}{l}\text { Azadirachta } \\
\text { indica }\end{array}$} & Leaf & 12 & 1 & 8.33 \\
\hline & Stem & 12 & 2 & 16.66 \\
\hline & Petiole & 15 & 0 & 0.00 \\
\hline
\end{tabular}

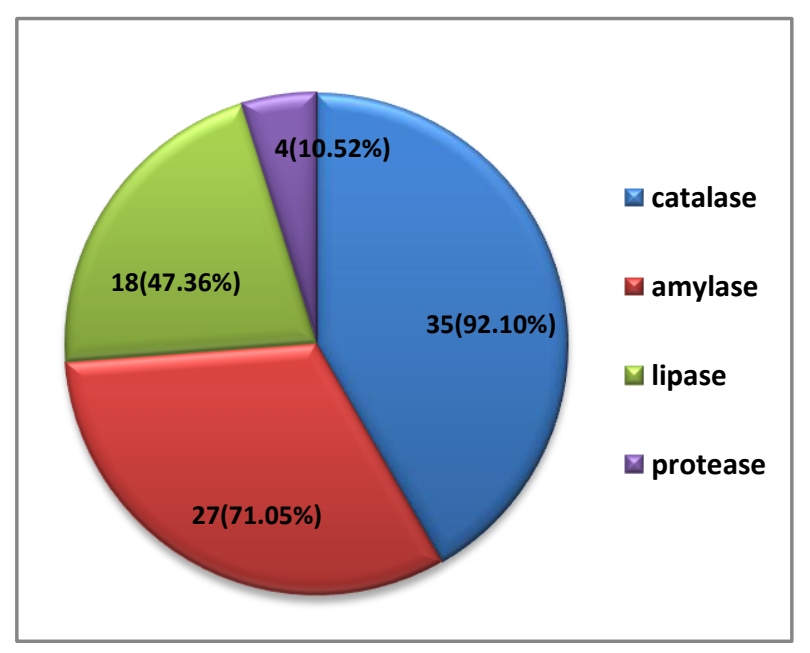

Figure 1 No. of Endophytic bacterial isolates showing positive activity for different enzymes 
Table 2 Phenotypic and biochemical characterization of endophytic bacteria

\begin{tabular}{|c|c|c|c|c|c|c|c|c|c|c|c|c|c|}
\hline Isolate & Grams stain & Shape & Glu & Lac & Suc & Mannitol & Motility & Citrate & $\mathrm{H}_{2} \mathrm{~S}$ & Acid & Gas & Indole & Urea \\
\hline AP01 & -ve & Rods & +ve & $+\mathrm{ve}$ & $+\mathrm{ve}$ & $+v e$ & $+v e$ & $+\mathrm{ve}$ & -ve & $+v e$ & -ve & -ve & -ve \\
\hline AS01 & -ve & Rods & $+v e$ & $+v e$ & $+\mathrm{ve}$ & $+\mathrm{ve}$ & $+\mathrm{ve}$ & -ve & -ve & $+\mathrm{ve}$ & $-\mathrm{ve}$ & -ve & $+\mathrm{ve}$ \\
\hline AS02 & $+\mathrm{ve}$ & Rods & $+\mathrm{ve}$ & $+\mathrm{ve}$ & $+\mathrm{ve}$ & $+\mathrm{ve}$ & $+\mathrm{ve}$ & -ve & -ve & $+\mathrm{ve}$ & -ve & $-v e$ & $-v e$ \\
\hline AL01 & -ve & Rods & $+\mathrm{ve}$ & $+\mathrm{ve}$ & $+\mathrm{ve}$ & $+\mathrm{ve}$ & $+\mathrm{ve}$ & $-v e$ & -ve & $+\mathrm{ve}$ & -ve & -ve & $+\mathrm{ve}$ \\
\hline OS01 & -ve & Rods & $+\mathrm{ve}$ & $+\mathrm{ve}$ & $+\mathrm{ve}$ & $+\mathrm{ve}$ & $+\mathrm{ve}$ & $+\mathrm{ve}$ & -ve & $+\mathrm{ve}$ & $+\mathrm{ve}$ & -ve & $+\mathrm{ve}$ \\
\hline OSO2 & -ve & Rods & $+\mathrm{ve}$ & $+\mathrm{ve}$ & $+\mathrm{ve}$ & $-\mathrm{ve}$ & -ve & -ve & -ve & $+\mathrm{ve}$ & +ve & -ve & $-\mathrm{ve}$ \\
\hline OS03 & -ve & Rods & $+\mathrm{ve}$ & $+\mathrm{ve}$ & $+\mathrm{ve}$ & $+\mathrm{ve}$ & $+\mathrm{ve}$ & $+\mathrm{ve}$ & -ve & $+\mathrm{ve}$ & $+\mathrm{ve}$ & $-v e$ & $+\mathrm{ve}$ \\
\hline OL01 & -ve & Rods & $+\mathrm{ve}$ & $+\mathrm{ve}$ & $+\mathrm{ve}$ & $+\mathrm{ve}$ & $+\mathrm{ve}$ & $+\mathrm{ve}$ & -ve & $+\mathrm{ve}$ & -ve & -ve & $+\mathrm{ve}$ \\
\hline OP01 & $+\mathrm{ve}$ & Rods & $+\mathrm{ve}$ & $+\mathrm{ve}$ & $+\mathrm{ve}$ & $+\mathrm{ve}$ & $+\mathrm{ve}$ & -ve & -ve & $+\mathrm{ve}$ & $+\mathrm{ve}$ & -ve & $+\mathrm{ve}$ \\
\hline OP02 & $+v e$ & Rods & $+\mathrm{ve}$ & $+\mathrm{ve}$ & $+\mathrm{ve}$ & $+v e$ & $+v e$ & $+\mathrm{ve}$ & -ve & $+\mathrm{ve}$ & -ve & -ve & -ve \\
\hline NS01 & $+v e$ & Rods & $+v e$ & $+v e$ & $+\mathrm{ve}$ & $+\mathrm{ve}$ & $+v e$ & -ve & -ve & $+\mathrm{ve}$ & -ve & -ve & -ve \\
\hline NS02 & -ve & Rods & $+v e$ & $+v e$ & $+\mathrm{ve}$ & -ve & -ve & $+\mathrm{ve}$ & -ve & $+\mathrm{ve}$ & -ve & -ve & -ve \\
\hline NL01 & $+v e$ & Cocci & $+v e$ & $+v e$ & $+\mathrm{ve}$ & -ve & -ve & -ve & -ve & $+v e$ & -ve & -ve & $+\mathrm{ve}$ \\
\hline CL01 & -ve & Rods & $+\mathrm{ve}$ & $+\mathrm{ve}$ & $+\mathrm{ve}$ & $+\mathrm{ve}$ & $+\mathrm{ve}$ & -ve & -ve & $+\mathrm{ve}$ & -ve & -ve & $-v e$ \\
\hline CL02 & -ve & Rods & $+\mathrm{ve}$ & $+v e$ & $+\mathrm{ve}$ & $+\mathrm{ve}$ & $+v e$ & $+v e$ & -ve & $+v e$ & -ve & -ve & -ve \\
\hline CLO3 & -ve & Rods & $+\mathrm{ve}$ & $+\mathrm{ve}$ & $+\mathrm{ve}$ & $+\mathrm{ve}$ & $+\mathrm{ve}$ & $+\mathrm{ve}$ & -ve & $+\mathrm{ve}$ & -ve & -ve & -ve \\
\hline CL04 & $+\mathrm{ve}$ & Cocci & $+v e$ & $+v e$ & $+v e$ & $+v e$ & -ve & $+v e$ & -ve & $+\mathrm{ve}$ & -ve & -ve & $+\mathrm{ve}$ \\
\hline CS01 & $+v e$ & Cocci & $+\mathrm{ve}$ & $+v e$ & $+\mathrm{ve}$ & $+v e$ & -ve & $+\mathrm{ve}$ & -ve & $+\mathrm{ve}$ & $-\mathrm{ve}$ & $-v e$ & -ve \\
\hline $\mathrm{CS} 02$ & -ve & Rods & $+v e$ & $+v e$ & $+v e$ & $+\mathrm{ve}$ & $+\mathrm{ve}$ & $+\mathrm{ve}$ & -ve & $+\mathrm{ve}$ & -ve & -ve & $+\mathrm{ve}$ \\
\hline $\mathrm{CS} 03$ & $-\mathrm{ve}$ & Rods & $+v e$ & $+v e$ & $+\mathrm{ve}$ & -ve & -ve & -ve & -ve & $+\mathrm{ve}$ & $-\mathrm{ve}$ & $-v e$ & $-v e$ \\
\hline $\mathrm{CS} 04$ & $+v e$ & Rods & $+v e$ & $+v e$ & $+\mathrm{ve}$ & $+v e$ & $+v e$ & $+v e$ & -ve & $+v e$ & $+v e$ & -ve & -ve \\
\hline CS05 & -ve & Rods & $+v e$ & $+v e$ & $+\mathrm{ve}$ & $+v e$ & -ve & -ve & -ve & $+v e$ & -ve & -ve & $+\mathrm{ve}$ \\
\hline CS06 & $+v e$ & Rods & $+v e$ & $+v e$ & $+\mathrm{ve}$ & $+v e$ & $+v e$ & $+v e$ & -ve & $+\mathrm{ve}$ & -ve & -ve & $+v e$ \\
\hline $\mathrm{CS} 07$ & $+v e$ & Rods & $+\mathrm{ve}$ & $+v e$ & $+\mathrm{ve}$ & $+\mathrm{ve}$ & $+v e$ & $+\mathrm{ve}$ & -ve & $+\mathrm{ve}$ & -ve & -ve & -ve \\
\hline CS08 & $+v e$ & Cocci & $+v e$ & $+v e$ & $+\mathrm{ve}$ & -ve & -ve & $+v e$ & -ve & $+v e$ & -ve & -ve & $+\mathrm{ve}$ \\
\hline CSO9 & $+v e$ & Cocci & $+\mathrm{ve}$ & $+v e$ & $+\mathrm{ve}$ & -ve & -ve & $+\mathrm{ve}$ & -ve & $+\mathrm{ve}$ & -ve & -ve & $+\mathrm{ve}$ \\
\hline CS10 & -ve & Rods & $+\mathrm{ve}$ & $+v e$ & $+\mathrm{ve}$ & $+\mathrm{ve}$ & $+v e$ & $+v e$ & -ve & $+v e$ & -ve & -ve & $+\mathrm{ve}$ \\
\hline CSLS11 & -ve & Rods & $+\mathrm{ve}$ & $+v e$ & $+\mathrm{ve}$ & -ve & -ve & $+\mathrm{ve}$ & -ve & $+\mathrm{ve}$ & -ve & -ve & -ve \\
\hline CSLS12 & -ve & Rods & $+\mathrm{ve}$ & $+\mathrm{ve}$ & $+\mathrm{ve}$ & $+\mathrm{ve}$ & $+\mathrm{ve}$ & $+\mathrm{ve}$ & -ve & $+\mathrm{ve}$ & +ve & $-v e$ & $+\mathrm{ve}$ \\
\hline CSLS13 & -ve & Rods & $+\mathrm{ve}$ & $+\mathrm{ve}$ & $+\mathrm{ve}$ & $+\mathrm{ve}$ & $+\mathrm{ve}$ & $+\mathrm{ve}$ & -ve & $+\mathrm{ve}$ & -ve & -ve & -ve \\
\hline CSLS14 & -ve & Rods & $+\mathrm{ve}$ & $+\mathrm{ve}$ & $+\mathrm{ve}$ & $+\mathrm{ve}$ & $+\mathrm{ve}$ & $+\mathrm{ve}$ & -ve & $+\mathrm{ve}$ & -ve & $-\mathrm{ve}$ & $+\mathrm{ve}$ \\
\hline CSLS15 & -ve & Rods & $+\mathrm{ve}$ & $+\mathrm{ve}$ & $+\mathrm{ve}$ & $+\mathrm{ve}$ & $+\mathrm{ve}$ & $+\mathrm{ve}$ & -ve & $+\mathrm{ve}$ & $+\mathrm{ve}$ & -ve & $+\mathrm{ve}$ \\
\hline CSLS16 & -ve & Rods & $+\mathrm{ve}$ & $+v e$ & $+\mathrm{ve}$ & $+\mathrm{ve}$ & $+\mathrm{ve}$ & $+\mathrm{ve}$ & -ve & $+\mathrm{ve}$ & -ve & -ve & -ve \\
\hline CSLS17 & -ve & Rods & $+\mathrm{ve}$ & $+v e$ & $+\mathrm{ve}$ & $+\mathrm{ve}$ & $+\mathrm{ve}$ & $+\mathrm{ve}$ & -ve & $+v e$ & -ve & -ve & -ve \\
\hline RSTS01 & -ve & Rods & $+\mathrm{ve}$ & $+\mathrm{ve}$ & $+\mathrm{ve}$ & $-\mathrm{ve}$ & -ve & $-v e$ & -ve & $+\mathrm{ve}$ & -ve & -ve & $+\mathrm{ve}$ \\
\hline RSTS02 & -ve & Rods & $+\mathrm{ve}$ & $+v e$ & $+\mathrm{ve}$ & -ve & -ve & $+\mathrm{ve}$ & -ve & $+\mathrm{ve}$ & -ve & -ve & $+\mathrm{ve}$ \\
\hline RSTS03 & -ve & Rods & $+\mathrm{ve}$ & $+\mathrm{ve}$ & $+\mathrm{ve}$ & -ve & -ve & $+\mathrm{ve}$ & -ve & $+\mathrm{ve}$ & -ve & -ve & $+\mathrm{ve}$ \\
\hline RSLS01 & $+\mathrm{ve}$ & Rods & $+\mathrm{ve}$ & +ve & $+\mathrm{ve}$ & $-\mathrm{ve}$ & -ve & $+\mathrm{ve}$ & -ve & $+\mathrm{ve}$ & -ve & -ve & $+\mathrm{ve}$ \\
\hline
\end{tabular}

Glu-glucose; Suc - sucrose; Lac-lactose; $\mathrm{H}_{2} \mathrm{~S}$ - Hydrogen Sulfide; +ve - positive, -ve-negative; Gm +ve Rods - Bacillus sp; Gm-ve Rods Pseudomonas sp; Gm +ve Cocci - Not identified; AP-Aegle Petiole; AS-Aegle Stem; AL-Aegle Leaf; OS-Ocimum Stem; OL-Ocimum Leaf; OP-Ocimum Petiole; NL- Neem Leaf; NS- Neem Stem ; CL-Calotropis Leaf; CS-Calotropis Stem; CSLS- Calotropis Stem (Longitudinal Stem); RSTS- Rawolfia Stem (Transverse Section); RSLS- Rawolfia Stem (Longitudinal Section) 
Table 3 Screening for antibacterial activity by the isolates

\begin{tabular}{|lccc|}
\hline Isolates & $\begin{array}{c}\text { Staphylococcus } \\
\text { aureus }\end{array}$ & $\begin{array}{c}\text { Pseudomonas } \\
\text { aeruginosa }\end{array}$ & $\begin{array}{c}\text { Escherichia } \\
\text { coli }\end{array}$ \\
\hline OL01 & +++ & + & +++ \\
\hline OS01 & + & + & +++ \\
\hline OS03 & ++ & +++ & +++ \\
\hline AL01 & ++ & - & - \\
\hline AP01 & - & - & ++ \\
\hline CL02 & + & - & ++ \\
\hline CL03 & + & - & ++ \\
\hline CS10 & + & - & ++ \\
\hline CSLS16 & - & + & + \\
\hline CSLS17 & - & - & + \\
\hline RSTS01 & + & + & + \\
\hline RSTS02 & + & + & + \\
\hline RSTS03 & + & + & - \\
\hline RSLS01 & + & + & + \\
\hline NS01 & ++ & + & + \\
\hline
\end{tabular}

+ positive (+ - zone size 5-10mm, ++ - zone size 11-15mm, +++ zone size 16-20mm); - negative; AP-Aegle Petiole; AL-Aegle Leaf; OS- Ocimum Stem; OL- Ocimum Leaf; NS- Neem Stem ; CLCalotropis Leaf; CS- Calotropis Stem; CSLS- Calotropis Stem (Longitudinal Stem); RSTS- Rawolfia Stem (Transverse Section); RSLS- Rawolfia Stem (Longitudinal Section)

\subsection{Determination of plant growth promoting traits}

All 38 endophytic isolates were characterized for PGP traits such as Indole Acetic Acid (IAA), siderophore, ammonia production and phosphate solubilization.

\subsubsection{Indole Acetic acid production}

All the isolates were screened for quantitative production of IAA, result of study revealed that, 8 isolates (OS01, AL01, OL01, OS03, CL02, CS10, CSLS16, RSLS01) were positive for IAA production. The isolate OS03 observed to produce highest IAA i.e., $17.715 \mathrm{ug} / \mathrm{ml}$ followed by OS01 $(16.833 \mathrm{ug} / \mathrm{ml})$ and OL01 $(15.710 \mathrm{ug} / \mathrm{ml})$ and the least IAA producer was found to be RSLS01(6.891ug/ml) shown in Table 4.

\subsubsection{Phosphate solubilization}

Results related to phosphate solubilizing activities on Pikovskaya's agar (PVKA) medium have been presented in Table 4. Results of study revealed that total six isolates viz., AP01,
OS01, OL01, OS03, CL02, CS10 have phosphate solubilization activity on PVKA medium. Isolate AP01showed maximum phosphate solubilizing index, indicating highest phosphatase activity in comparison to other isolates while the minimum phosphate solubilization activity was reported in case of isolate CS10.

\subsubsection{Siderophore production}

Out of 38 endophytic bacteria, 12 (AP01, OS01, OL01, OS03, NSB001, CL02, CL03, CS10, CSLS16, RSTS01, RSTS02, RSTS03) isolates were positive for siderophore production. These isolates have the efficiency to produce siderophore, which chellates iron from the medium. Maximum siderophore production index (SI) was found to be 2.75 by OS03 and the least SI was observed in case of RSTS03 with SI 2.14 shown in Table 4.

\subsubsection{Ammonia production}

When all the isolates were subjected for ammonia production test, it was observed that only 8 isolates (AP01, OS01, OL01, OS03, CS04, CS07, CS10, RSLS01) were positive for ammonia production (Table 4).

Based on comparative plant growth promoting activities, it was observed that four isolates (OS01, OL01, OS03, CS10) were positive for all the above-mentioned Plant Growth Promotion traits showing their efficiency to promote plant growth.

\section{Discussion}

The importance of endophytic bacteria is well known since long time but recently it gets more concern of research community because these play specific role in protecting the host plants against insect diseases, and performing plant growth promoting activities through mineralization and plant growth hormone production (Strobel \& Long 1998). The diversity of the endophytes largely depends upon the isolation of endophytes (Das et al., 2007) and the isolation of endophytes ensures proper surface sterilization so that epiphytic microbes were completely removed (Hallmann et al., 1997). The surface sterilization process as described in this experiment was satisfactory as no growth appeared on the control plate and all the isolates were considered to be of endophytic origin.

Novel bioactive metabolites are isolated from bacterial and fungal endophytic isolates from medicinal plants possess antimicrobial activity (Tayung \& Jha, 2006). Antibacterial activities of 15 endophytic isolates against $S$. aureus, $P$. aeruginosa and E. coli were observed during this investigation, is indicative of their bioactive potential. Since, the antibacterial activity of the isolates were observed on solid nutrient agar plates, through disc diffusion method, it is suggested that the antimicrobial components produced by these isolates to be extracellular and water soluble. 
Table 4 Plant growth promoting activity by the endophytic bacterial isolates

\begin{tabular}{|c|c|c|c|c|c|c|c|}
\hline $\begin{array}{l}\text { Bacterial } \\
\text { isolates }\end{array}$ & $\begin{array}{c}\text { IAA } \\
\text { production }\end{array}$ & IAA(ug/ml) & $\begin{array}{c}\text { Phosphate } \\
\text { solubilization }\end{array}$ & PSI & $\begin{array}{c}\text { Siderophore } \\
\text { production }\end{array}$ & SI & $\begin{array}{c}\text { Ammonia } \\
\text { production }\end{array}$ \\
\hline AP01 & - & - & + & 3.18 & + & 2.39 & + \\
\hline AS01 & - & - & - & - & - & - & - \\
\hline AS02 & - & - & - & - & - & - & - \\
\hline AL01 & + & 13.132 & - & - & - & - & - \\
\hline OS01 & + & 16.833 & + & 2.30 & + & 2.47 & + \\
\hline OS02 & - & - & - & - & - & - & - \\
\hline OS03 & + & 17.715 & + & 2.44 & + & 2.75 & + \\
\hline OL01 & + & 15.710 & + & 2.20 & + & 2.60 & + \\
\hline OP01 & - & - & - & - & - & - & - \\
\hline OP02 & - & - & - & - & - & - & - \\
\hline NS01 & - & - & - & - & + & 2.16 & - \\
\hline NS02 & - & - & - & - & - & - & - \\
\hline NL01 & - & - & - & - & - & - & - \\
\hline CL01 & - & - & - & - & - & - & - \\
\hline CLO2 & + & 8.731 & + & 2.36 & + & 2.50 & - \\
\hline CL03 & - & - & - & - & + & 2.42 & - \\
\hline CL04 & - & - & - & - & - & - & - \\
\hline CS01 & - & - & - & - & - & - & - \\
\hline $\mathrm{CS} 02$ & - & - & - & - & - & - & - \\
\hline $\mathrm{CS} 03$ & - & - & - & - & - & - & + \\
\hline CS04 & - & - & - & - & - & - & - \\
\hline CS05 & - & - & - & - & - & - & - \\
\hline CS06 & - & - & - & - & - & - & - \\
\hline CS07 & - & - & - & - & - & - & + \\
\hline CS08 & - & - & - & - & - & - & - \\
\hline CS09 & - & - & - & - & - & - & - \\
\hline CS10 & + & 10.346 & + & 2.12 & + & 2.42 & + \\
\hline CSLS11 & - & - & - & - & - & - & - \\
\hline CSLS12 & - & - & - & - & - & - & - \\
\hline CSLS13 & - & - & - & - & - & - & - \\
\hline CSLS14 & - & - & - & - & - & - & - \\
\hline CSLS15 & - & - & - & - & - & - & - \\
\hline CSLS16 & + & 8.923 & - & - & + & 2.26 & - \\
\hline CSLS17 & - & - & - & - & - & - & - \\
\hline RSTS01 & - & - & - & - & + & 2.57 & - \\
\hline RSTS02 & - & - & - & - & + & 2.16 & - \\
\hline RSTS03 & - & - & - & - & + & 2.14 & - \\
\hline RSLS01 & + & 6.891 & - & - & - & - & + \\
\hline
\end{tabular}

The hydrolytic enzymes secreted by the bacteria play an important role in suppression of pathogens which aids to the defence mechanism of the host plant (Buchenauer, 1998). In the present study maximum numbers of isolates were found positive for catalase and amylase activities followed by lipase and protease, indicating their capabilities to protect the host from pathogen attack.

Furthermore, during the present study, $21.05 \%$ of the isolates produced ammonia, by hydrolyzing urea. Microorganism produce ammonia as secondary metabolite by hydrolyzing urea into ammonia and $\mathrm{CO}_{2}$, leads to satisfy the nitrogen demand of the host plant and also it helps in reducing the colonization of pathogens (Mbai et al. 2013). The production of ammonia is another characteristic of PGPR that indirectly influence development of plant (Ngoma et al., 2014). Also help in accumulation, synthesis and supply of nitrogen to the host which leads to promote root and shoot elongation resulting in increase of plant biomass (Marques et al., 2010).

Direct promotion induced by PGPB aids to the plant growth and development by production of phytohormones like Indole-3acetic acid (IAA), Gibberellic acid (GA3), nitrogen fixation, siderophore production (Glick et al., 1999) and phosphate solubilization (de-Bashan \& Bashan, 2004).

Endophytes have also been shown to promote plant growth by producing phytohormones like IAA (Mendes et al., 2007), which not only increase the root size also results in greater nutrient absorption $(\mathrm{Li}$ et al., 2008). Several reports such as Lee et al. (2004) and Mutluru \& Konada (2007) corroborates with our findings indicating increase of IAA production while the medium supplemented with L-Tryptophan as observed in present investigation. Datta \& Basu (2000) suggested that IAA production is the characteristic of gram -ve bacteria in contrast to these results, findings of present study suggested that both gram +ve and gram -ve isolates producing IAA. However, Wahyudi et al. (2011) reported IAA production by gram +ve bacteria. 
Siderophore production by soil bacteria, results in uptake of Fe, $\mathrm{Zn}, \mathrm{Cu}$ etc. by plants (Gururani et al., 2012). In addition, production of antimicrobial compounds is directly induced by siderophore production ability of bacteria (Joseph et al., 2007; Wahyudi et al., 2011). Results of present study are in agreement of the above said findings and 12 isolates were positive for siderophore production which could be attributable to the plant growth promoting activities as well as antibacterial activities against the three test pathogens as recorded during this investigation. Siderophore production by associative endophytic microorganisms, help in colonization of bacteria to the host tissue from the rhizospheric zone (Loaces et al., 2011 ). Sessitsch et al. (2012), reported siderophore producing endophytic bacterial community isolated from rice roots, acts as biocontrol agent against other pathogens. The PGPB isolates with high siderophore producing activity can be further studied for its ability to confer disease resistance in higher plants.

Soil bacteria are capable to solubilize insoluble phosphate and make it available to plant (Ahemad \& Kibret, 2014) and this activity is highly important as these microorganisms acts as biofertilizers promoting plant growth. Five isolates(OS01, OS03, OL01, CL02 \& CS10) showed medium PSI index (2-3) whereas, one isolate (AP01) showed high PSI (3.18) during our study while studied on Pikovaskaya's Agar as per the classification of Silva \& Vidor (2000), could find their use as phosphate biofertilizers after proper scientific studies.

\section{Conclusion}

Through this investigation we reported, the isolation of culturable endophytic bacteria from six medicinal plants belonging to both Gram positive and Gram negative forms. Our isolates showed plant growth promoting activities like IAA production, Phosphate solubilization and siderophore production. Further, scientific investigation of these isolates in these lines are necessary to exploit potential of these isolates, as plant growth promoting microorganisms in order to enjoy the benefits of this preliminary scientific investigation.

\section{Acknowledgement}

The authors are thankful to Science and Technology Department, Govt. of Odisha for financial support. The authors are also thankful to the HoD, Department of Botany, and Director, College of Basic Science and Humanities, Orissa University of Agriculture and Technology (OUAT), for providing the laboratory facilities.

\section{Conflict of interest}

All the authors declare that there is no conflict of interest.

\section{References}

Ahemad M, Kibret M (2014) Mechanisms and applications of plant growth promoting rhizobacteria: current perspective. Journal of King Saud University Science 26:1-20.

Amaresan N, Jayakumar V, Thajuddin N (2014) Isolation and characterization of endophytic bacteria associated with chilli (Capsicum апnиит) grown in coastal agricultural ecosystem. Indian Journal of Biotechnology 13:247- 255.

Andreote FD, Gumiere T, Durrer A (2014) Exploring interactions of plant microbiomes. Scientia Agrícola 71:528-539.

Azevedo JL, Maccheroni Jr W, Pereira JO, Araújo de WL (2000) Endophytic microorganisms: a review on insect control and recent advances on tropical plants. Electronic Journal of Biotechnology 3:15-16.

Buchenauer H (1998) Biological control of soil-borne diseases by rhizobacteria. Journal of Plant Diseases and Protection 105:329-348.

Cappuccino JG, Sherman N (2002) Microbiology: A laboratory manual (8th edition), Pearson. ISBN-13: 978-0805325782.

Collins CH, Lyne PM (1970) Microbiological methods. $6^{\text {th }}$ Edn. London ; Butterworth

Compant S, Reiter B, Sessitsch A, Nowak J, Clément C, Ait Barka E (2005) Endophytic colonization of Vitis vinifera L. by a plant growth-promoting bacterium, Burkholderia sp. strain PsJN. Applied and Environmental Microbiology 71:1685-1693.

Das M, Royer TV, Leff LG (2007) Diversity of fungi, bacteria and actinomycetes on leaves decomposing in a stream. Applied and Environmental Microbiology 73:756-767.

Datta C, Basu P (2000) lndole acetic acid production by a Rhizobium species from root nodules of a leguminous shrub Cajanus cajan. Microbiological Research 155:123-127.

De-Bashan LE, Bashan Y (2004) Recent advances in removing phosphorus from waste water and its future use as fertilizer (1997-2003). Water research 38:4222-4246.

Germaine K, Keogh E, Garcia-Cabellos G, Borremans B, Van Der Lelie D, Barac T, Campbell CD (2004) Colonisation of poplar trees by gfp expressing bacterial endophytes. FEMS Microbiology Ecology 48:109-118.

Glick BR, Patten CL, Holguin G, Penrose DM (1999) Biochemical and genetic mechanisms used by plant growth promoting bacteria. Imperical college press, London, Pp.187-189.

Journal of Experimental Biology and Agricultural Sciences http://www.jebas.org 
Gunatilaka AAL (2006) Natural Products from plant associated microorganisms: distribution, structural diversity, bioactivity and implications of their occurrence. Journal of Natural Products 69:3:509-26.

Gururani MA, Upadhyaya CP, Baskar V, Venkatesh J, Nookaraju A, Park SW (2012) Plant growth-promoting rhizobacteria enhance abiotic stress tolerance in Solanum tuberosum through inducing changes in the expression of ROS-scavenging enzymes and improved photosynthetic performance. Journal of Plant Growth Regulation 32:245-258.

Hallmann J, Quadt-Hallmann A, Mahaffee WF, Kloepper JW (1997) Bacterial endophytes in agricultural crops. Canadian Journal of Microbiology 43:895-914.

Holt JG, Krieg NR, Sneath PA, Staley JT, Williams ST (1994) Bergey's Manual of determinate bacteriology.

Jena S, Rath CC (2016) Effect of Non-Organophosphate pesticides on phosphatase activity of a soil fungus Aspergillus awamori Kc316117 isolated from a moist deciduous forest in Odisha, India. Journal of Advanced Microbiology $2: 330-336$.

Joseph B, Patra RR, Lawrence R (2007) Characterization of plant growth promoting rhizobacteria associated with chickpea (Cicer arietinum L.). International Journal of Plant Production 2:141-1520.

Kloepper JW, Ryu CM (2006) Bacterial endophytes as elicitors of induced systemic resistance. In Microbial root endophytes (33-52), Springer, Berlin.

Kumar A, Singh R, Yadav A, Giri DD, Singh PK, Pandey KD (2016) Isolation and characterization of bacterial endophytes of Curcuma longa L. 3 Biotech 6:60.

Lee S, Flores-Encarnacion M, Contreras-Zentella M, Garcia-Flores L, Escamilla JE, Kennedy C (2004) Indole-3-acetic acid biosynthesis is deficient in Gluconacetobacter diazotrophicus strains with mutations in cytochrome $\mathrm{C}$ biogenesis genes. Journal of Bacteriology 186: 5384-539.

Li JH, Wang ET, Chen WF, Chen WX (2008) Genetic diversity and potential for promotion of plant growth detected in nodule endophytic bacteria of soybean grown in Heilongjiang province of China. Soil Biology and Biochemistry 40:238-246.

Loaces I, Lucía F, Ana FS (2011) Dynamics, diversity and function of endophytic siderophore-producing bacteria in rice. Microbial Ecology 61:606-618.

Lodewyckx C, Vangronsveld J, Porteous F, Moore ER, Taghavi S, Mezgeay M, der Lelie DV (2002) Endophytic bacteria and their potential applications. Critical reviews in plant sciences 21:583-606.
Marques APGC, Pires C, Moreira H, Rangel AOSS, Castro PML (2010) Assessment of the plant growth promotion abilities of six bacterial isolates using Zea mays as indicator plant. Soil Biology and Biochemistry 42:1229-1235.

Mastretta C, Barac T, Vangronsveld J, Newman L, Taghavi S, Lelie DVD (2006) Endophytic bacteria and their potential application to improve the phytoremediation of contaminated environments. Biotechnology and Genetic Engineering Reviews 23:175-188.

Mbai FN, Magiri EN, Matiru VN, Nganga J, Nyambati VCS (2013) Isolation and characterization of bacterial root endophytes with potential to enhance plant growth from Kenyan Basmati rice. American International Journal of Contemporary Research 3:25-40.

Mendes R, Pizzirani-Kleiner AA, Araujo WL, Raaijmakers JM (2007) Diversity of cultivated endophytic bacteria from sugarcane: genetic and biochemical characterization of Burkholderia cepacia complex isolates. Applied and Environmental Microbiology 73:7259-7267.

Mutluru S, Konada VM (2007) Bioproduction of indole acetic acid by Rhizobium strains isolated from root nodules of green manure crop, Sesbania sesban (L.). Iranian Journal of Biotechnology 53:178-182.

Ngoma L, Mogatlanyan K, Babalola OO (2014) Screening of endophytic bacteria towards the development of cottage industry: An in vitro study. Journal of Human Ecology 47: 45-36.

Patten CL, Glick BR (2002) The role of Pseudomonas putida indole acetic acid in the development of the host plant root system. Applied and Environmental Microbiology 68:3795-3801.

Pavlo A, Leonid O, Iryna Z, Natalia K, Maria PA (2011) Endophytic bacteria enhancing growth and disease resistance of potato (Solanum tuberosum L.). Biological Control 56:43-49.

Rashid S, Charles TC, Glick BR (2012) Isolation and characterization of new plant growth-promoting bacterial endophytes. Applied Soil Ecology 61:217-224.

Sessitsch A, Hardoim PJ, Döring WA (2012) Functional characteristics of an endophyte community colonizing rice roots as revealed by metagenomic analysis. Molecular Plant Microbe Interactions 25:28- 36 .

Silva Filho GN, Vidor C (2000) Phosphate solubilization by microorganisms in the presence of different carbon source. Revista Brasileira de Ciência do Solo 24:311-319. 
Strobel G, Daisy B, Castillo U, Harper J (2004) Natural products from endophytic microorganisms. Journal of Natural Products 67:257-68.

Strobel GA, Long DM (1998) Endophytic microbes embody pharmaceutical potential. American Society for Microbiology 64: 263-268.

Taghavi S, Garafola C, Monchy S, Newman L, Hoffman A, Weyens N, Barac T, Vangronsveld J, Vander Lelie D (2009) Genome survey and characterization of endophytic bacteria exhibiting a beneficial effect on growth and development of poplar trees. Applied and Environmental Microbiology 75:748-75.
Tayung K, Jha DK (2006) Antimicrobial evaluation of some fungal endophytes isolated from the bark of Himalayan yew. World Journal of Agriculture Science 2:489-494.

Wahyudi AT, Astuti RP, Widyawati A, Meryandini A, Nawangsih AA (2011) Characterization of Bacillus sp. strains isolated from rhizosphere of soybean plants for their use as potential plant growth promoting Rhizobacteria. Journal of Microbiology and Antimicrobial Agents 3:34-40.

Wakelin SA, Warren RA, Harvey PR, Ryder MH (2004) Phosphate solubilization by Penicillium spp. closely associated with wheat roots. Biology and Fertility of Soils 40:36-43. 